\title{
The Design of Digital Television Multimedia Message Service System
}

\author{
Chun Jin \\ ChongQing University of Posts and Telecommunications \\ ChongQing JINOU Science \& Technology Co., LTD \\ Fan Yang \\ Chongqing University of Posts and Telecommunications \\ PO box 78 \#, Chongqing, 400065 China \\ E-mail: yangfanan1001@hotmail.com \\ Baohong Wan \\ ChongQing JINOU Science \& Technology Co., LTD \\ Xiaojun Zhou \\ ChongQing JINOU Science \& Technology Co., LTD
}

\begin{abstract}
This dissertation studies the Multimedia Message Services that based on the digital television system, and it is called TVMMS for short. The author discusses the TVMMS architecture, and specifies the address number distributed for every entity and addressing scheme. The TVMMS can perform the following functions: point to point communication between STB, communication between STB (Set Top Box) and VASP (Value Added Services Provider), communication between STB and mobile telephone, communication between STB and TV station. So, TVMMS will greatly enrich the application of digital television.
\end{abstract}

Keywords: Digital Television, Multimedia Message, TVMMS

\section{Introduction}

The operation of information industry mainly depends on the three supported networks which are telecommunication network, the Cable TV network and Internet. As far as China is concerned, in fact, it has two networks which are telecommunication network and the Cable TV network. At present, the telecommunications networks and the Cable TV network are basically independent of each other. We can't communicate between the two operations systems. But the United States and Britain in the 1990s allowed telecommunications operators and cable TV operators to enter the market of each other. And then they can communicate between the two operations systems. In China, the communication between the two operations systems is one of the most important solutions in the convergence of three networks. The digital television multimedia message service system we researched can achieve the convergence of the three networks in the application layer.

At present, the main factors restricting the development of Digital Television are lacking of funds and there are not the authorized user, and are lacking of content. The system this paper designed can achieve interoperability between Cable TV network, the Internet and telecommunications networks. The abundant contents on the Internet and telecommunications networks will be presented on the Television through this system. According to statistics, there are currently approximately 20,000 SPs (Service Provider). They provide a lot of contents for value added services. Similarly, with the adoption of this system, these Service Providers can access to the Cable TV networks and provide abundant contents to the Digital Television users. Thereby, it promotes the development of the Digital Television.

Through the Digital Television Multimedia Message Service System, the user can receive the multimedia message which sends from Internet and mobile phone. If there is a return channel, subscribers can also send message to Internet, mobile phone and television station. So this will greatly enhance the level of domestic information. And it contributes to achieve convergence of three networks in the application layer.

\section{TVMMS Reference Architecture (Note 1)}

\subsection{TVMMS in MMSE}

Figure 1 shows a unified Multimedia Message Service Environment (MMSE, Multimedia Messaging Service Environment). It includes Digital Television Multimedia Message Service TVMMS system; the mobile communications 
networks MMS system, the Public Switched Telephone Network (PSTN), Internet and other MMS systems. The MMSE provides all the necessary service elements, e.g. delivery, storage and notification functionality. These service elements may be located on one MMS system or be distributed across several different MMS systems. This MMSE makes these Multimedia Message systems which distributed across several different networks interoperability.

\subsection{TVMMS Reference Architecture}

Figure 2 shows the TVMMS system architecture. The function entity/module which is composed of the TVMMS system and the interface between them are listed below

\subsection{TVMMS Function Entity and Interfaces}

UA (User Agent): User Agent is the application layer's software which located on the STB. It provides the function of supporting for the TVMMS service. The user can receive, watch, write and send Digital Television Multimedia Message through the user agent. The TVMMS User Agent shall provide the following application layer functionalities. ( Note 2)

- The recipient of the message from TVMMS Relay/Server, including multimedia message and notification message;

- Presentation of the multimedia message to the user;

- Multimedia Message composition;

- Submission of the multimedia message to the TVMMS Relay/Server;

- Decryption and encryption of a MM;

- Storing of the message into STB;

- Management of the message store in STB.

TVMMS Relay/Server: The MMS Relay/Server is responsible for storage and notification, reports, and general handling of messages. The TVMMS Relay/Server is in the core position in the whole TVMMS system. The TVMMS Relay/Server element can be integrated together, also can be separated into two elements. They can communicate through MM2 interface. The TVMMS Relay/Server shall provide the following functionalities:

- Receiving, sending and forwarding the multimedia message;

- Conversion of the message format between the TVMMS Relay/Server and the External Server (e.g. Email Server) or between TVMMS Relay/Server and the other systems;

- Multimedia Message notification to the TVMMS User Agent;

- Routing forward Multimedia Message to the different TVMMS Relay/Servers;

- Generating and routing forward the read-reply reports;

- Analyzing addresses of messages, and routing forward the message according to the addresses information;

- Temporary storage of the TVMMS messages;

- Ensuring that messages are not lost until successfully delivered to another MMSE element.

- Supporting for DRM(Digital Right Management);

- Generating charging data records (CDR);

- Filtering the message according to the user information;

- Checking the estate of the STB; e.g. opening the STB and closing the STB. Supporting TVMMS message or not. If there are returned channel.

External Server: External Server, for example Email Server etc, can implement "television mail" function. We can send normal email to the specified television. External Server didn't belong to the functional modules which TVMMS need to have.

Management and Control: The Equipment of management and control is responsible for the management and control function of TVMMS System, such as system parameter configuration, addressing, routing, charging parameters, the control of user jurisdiction and the operation and maintenance of the TVMMS System.

Billing System: The billing system shall bill the users and VASP according to the CDR that the TVMMS Relay/Server generates.

Electronic Program Guide(EPG): EPG is an on-screen guide to scheduled broadcast television or radio programs, allowing a viewer to navigate, select, and discover content by time, title, channel, genre, etc.

TVMMS Message Storage Center: TVMMS is a storing and forwarding system. Upon the TVMMS Relay/Server has received the message, it caches the message and checks the address information. If the recipient User Agent located on 
its range, the TVMMS Relay/Server will send this message to the TVMMS message storage center. Then the TVMMS message storage center returned a URI of this message to the TVMMS Relay/Server. The TVMMS Relay/Server notified recipient User Agent there are new messages arrived (this notification include how to fetch the information of this TVMMS message).The TVMMS message storage center deleted this information (or deleted after a certain period of time), if the users receive this message successfully. If the users didn't download this message during a period of time, the TVMMS storage center also deleted the message. If the recipient User Agent didn't locate on its range, it needs forward the message to the recipient User Agent which located on the TVMMS Relay/Server according to the routing information. After received the correct response information, the TVMMS Relay/Server will delete the massage.

TVMMS user databases: The TVMMS user database shall provide:

- Management of the TVMMS user subscription information;

- Management of the user access control information;

- Set of rules how to handle incoming messages and their delivery;

TVMMS VASP: The TVMMS Value Added Services Provider shall provide value added services to the TVMMS users, such as music, TV cards etc. The TVMMS VASP connected with the TVMMS Relay/Server through the MM9 interface.

The interfaces in the TVMMS reference architecture are:

MM1: the reference point between the TVMMS User Agent and the TVMMS Relay/Server;

MM2: the reference point between TVMMS Relay and TVMMS Server. In specific implementation, Relay can be integrated with Server into a same physical device;

MM3: reference point between TVMMS Relay/Server and External Servers;

MM4: reference point between TVMMS Relay/Server and management control Server;

MM5: reference point between TVMMS Relay/Server and a billing system;

MM6: reference point between TVMMS Relay/Server and message store center;

MM7: reference point between TVMMS Relay/Server and user databases;

MM8: reference point between TVMMS Relay/Server and another TVMMS Relay/Server;

MM9: the reference point between TVMMS Relay/Server and VASP (Value Added Services Provider).

MM10: reference point between TVMMS Relay/Server and Electronic Program Guide(EPG);

\section{Conclusion}

The development of digital television and alteration of the transmission network is the important revolution of the Radio and Television system. It will change the people's live style in a way. The commercial success of digital television multimedia message service system discussed in this paper will greatly enhance the information level of the family and societies, reach the service application of digital television. And it is conducive to the development of digital TV terminals as a family information center. In China, the Cable TV network, Internet and telephone network are independent each other. It contributes to achieve convergence of three networks in the application of services.

\section{References}

3GPP TS 23.140v6.11.1: Technical Specification Group Core Network and Terminals, Multimedia Messaging Service, www.3gpp.org.

3GPP TS 22.140v6.7.0: Technical Specification Group Services and System Aspects, Multimedia Messaging Service, www.3gpp.org

ARCIDIACONO, A.: 'Multimedia services and data broadcasting via satellite', ibid., pp. 33-37

DRURY, G. M.: 'DVB channel coding standards for broadcasting compressed video services', Electvon. \&Commun. Eng.J., February 1997,9, (I), pp.11-20

ETS300429:Digital broadcasting Systems for television, Sound and data services; Framing structure, Channel Coding and modulation for Cable system(DVB-C spec)

ISO/IEC JTC 1/SC 29 11172-3 Coding of Moving pictures and Associated Audio for Digital Storage Media at up to about $1.5 \mathrm{Mbit} / \mathrm{s} .1993$

ISO/IEC 14496-1 Coding of audio-visual objects-Part1: Systems

ISO/IEC 14496-1 Coding of audio-visual objects-Part4: Conformance Testing

WAP-205-MMSArchOverview-20010425-a, http://www.wapforum.org 


\section{Notes}

Note 1 . This is an example for note 1.

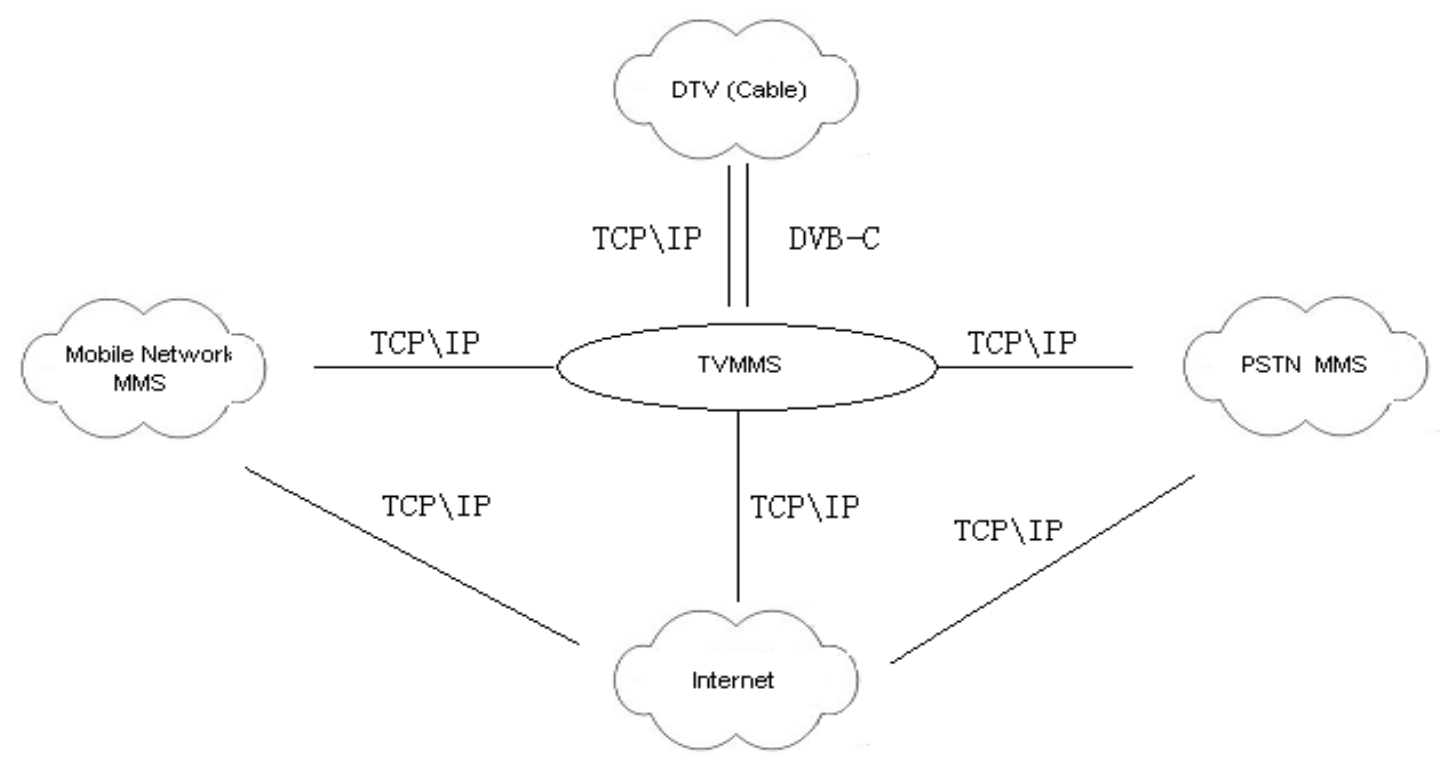

Figure 1. TVMMS in MMSE

Note 2. This is an example for note 2.

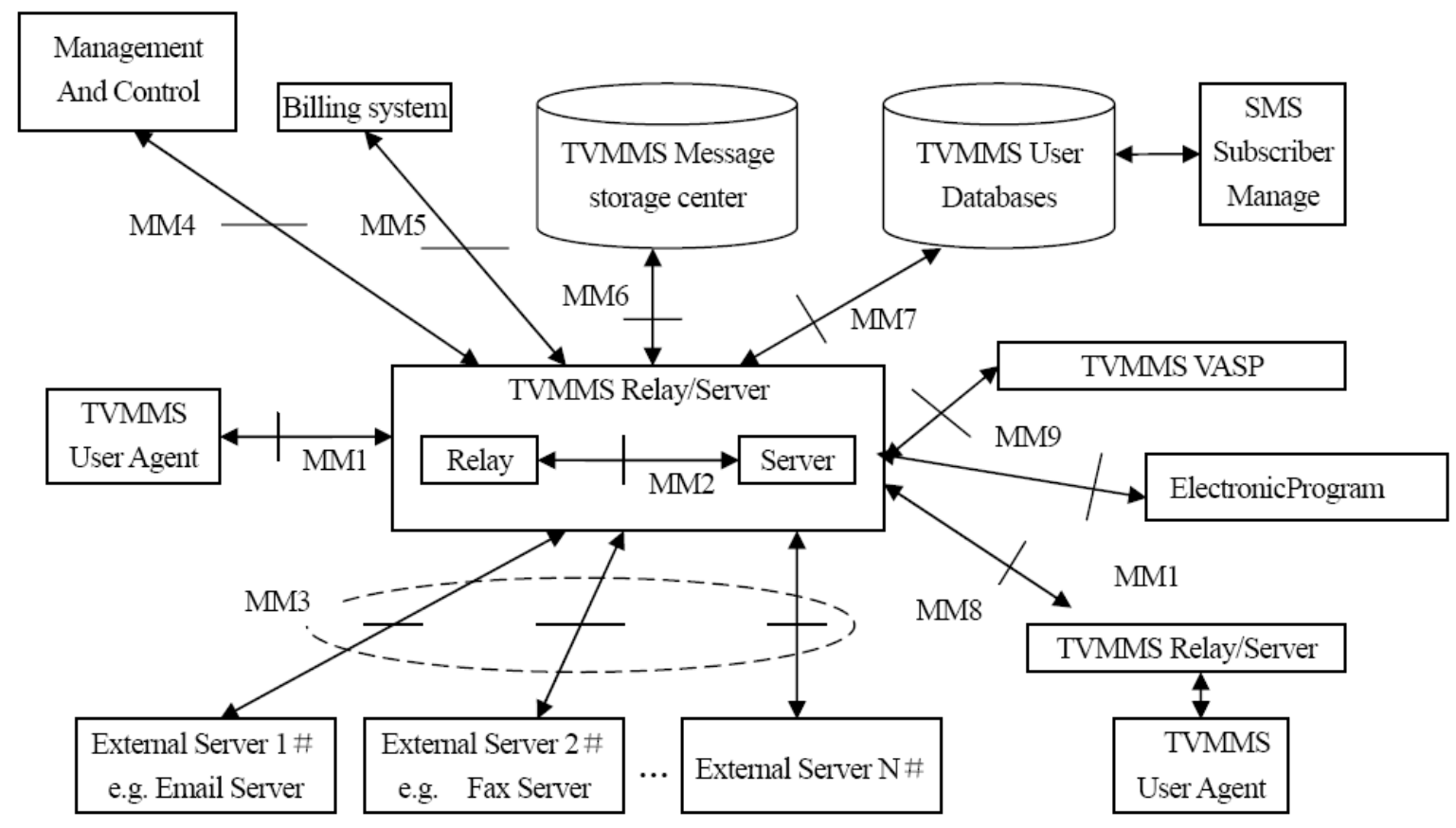

Figure 2. TVMMS reference architecture 